\title{
Research on the Application of Neural net in the Civil Engineering
}

\section{Semi-active Control}

\author{
Xianglan Li and Li Guo \\ Nanchang Key Laboratory of material and structure detection Jiangxi University of Technology
}

Keywords: Civil engineering; Semi-active control; Radial basis function neural network (RBFNN); Magneto-rheological damper/MR damper

\begin{abstract}
Earthquake poses serious hazard to human life and property security. Civil engineering structural vibration control especially the semi-active control can effectively reduce the earthquake damage to architecture. Neural network control has the ability of self-learning, adaptivity and parallel distributed processing capacity, and it is a new kind of control method. Especially the radial basis function neural network (RBFNN) has the feature of fast convergence rate and high precision, so the application of RBFNN in semi-active control of civil engineering structure has great significance to damping. The paper applied the RBFNN in the semi-active control of civil engineering structure, designed neutral network controller, and made simulated and comparative analysis on the damping effect.
\end{abstract}

\section{Introduction}

Earthquake poses serious hazard to human life and property security. In recent years, with the rapid development of modern society, the material standard of living has been greatly improved, and the city scale and quantity are also constantly expanding. The high-density population in the city will lead to terrible casualties and serious economic losses once the earthquake occurs. The earthquake has become increasingly frequent in recent years, and it seriously threatens the safety of people. China is one of the earthquake countries in the world, as it is in the position of Pacific seismic zone and Eurasia seismic zone, which are the most active one. For example, the "5-12" Wenchuan earthquake in 2008, had been the most destructive and most widespread earthquake since the founding of new China, and it caused huge property losses and casualties.

With the progress and development of modern society, people have stricter requirements for the safety and applicability of the designed architecture. Therefore, the main topic of the field of civil engineering structure is to study the new anti-seismic methods that are safer, reliable and economic. The structural vibration control theory developed in recent 20 years is one of the better anti-seismic methods in solving the problems, and has great practical significance in effectively reducing the disaster caused by the earthquake. In the early 1970s, some scholars began to set the control device on the building structure in order to reduce the vibration response of structures, and this is the structure vibration control in civil engineering. Compared with the traditional anti-seismic structure, the effect of damping capacity of structure vibration control is very obvious, which can reduce the seismic response of architecture to the range of quarter to one sixth. Structure vibration control has the advantage of safety, wide applicability and economy, which provides a new method for anti-seismic of civil engineering structure. 
Vibration control of civil engineering structure is divided into passive control, active control, hybrid control and semi-active control, and the research and application of the four methods have great development. Among them, the semi-active control method not only possesses the advantage of passive control reliability, but also has the advantage of strong adaptability of active control. The control device of semi-active control acquires control force by following building structure action; the small amount of energy input can achieve almost the same effect as active control. It is a kind of parameter control, and the change of parameter of semi-active control device can achieve adjusted control of building structure. The structure is simple and stable, and the control effect can be achieved by combining certain control algorithm. Therefore, the semi-active control has caused extensive attention of scholars both at home and abroad, and become a new focus on the international research field. This paper studies the semi-active control of civil engineering structure.

The study on semi-active control of civil engineering structure includes control device and control algorithm, and the study of these two aspects have achieved very good results. Magnetorheological fluid damper is an effective damping control device of civil engineering structure semi-active control. Any kind of control including the semi-active control, the control algorithm is the core content. The dynamic model of control object of semi-active control (building structure) and control device is very complex; therefore, the design of semi-active control algorithm has the certain difficulty, and classical control theory is based on the control of accurate model; the established model has simplified the actual structure, so the control effect will be greatly reduced. Intelligent control theory especially the development and application of neural network has brought convenience to the research on semi-active control. Intelligent control does not need precise structure calculation model; simple adjustment and strong learning ability have become the hotspot of the study on semi-active control of civil engineering structure. Neural network has brought new ideas to the semi-active control, and has great significance to reducing earthquake damage and improving the seismic resistance of building structure.

\section{The development of civil engineering structure vibration control}

Civil engineering structure vibration control is to set the control device in certain parts of the building structure; when the vibration response of building structure occurs, a set of control force will passively or actively be imposed on the building structure, or the dynamic characteristics of building structure will be changed so as to reduce the vibration response of building structure and meet the requirement of higher security and function. Structural vibration control is to improve the ability of resisting earthquake for building structure, improve the stability of the building structure when the earthquake struck, and meet the requirements of safety and applicability. Civil engineering structure vibration control integrated control theory, computer, machinery, and new material science; it is a new, comprehensive and multidisciplinary cross subject; it is the high-tech field of civil engineering, and is still in the preliminary stages of development and application. Structural vibration control has become the frontier and hot topics of the study on damping and seismic resistance to building structure, and attracted more and more scholars to research the structural vibration control.

According to whether rely on the input angle of external energy, the civil engineering structure vibration control can be divided into four kinds: passive control, active control, hybrid control and semi-active control. 


\section{Semi-active control}

The semi-active control of civil engineering structure is developed by active structural vibration control, and the principle block diagram is shown in Fig. 1. The control process of semi-active control depends on the vibration response of structures and earthquake excitation; the stiffness or damping parameters of semi-active control device can achieve real-time change by few external energy so as to reduce the vibration response of building structure, and the building structure can achieve real-time and adjustable control. The semi-active control is a kind of parameter control; according to the vibration response, directly adjust the parameters of the actuator can achieve good damping effect. Up to now, in structural vibration control method, the semi-active control is most promising and has highest cost performance in civil engineering application

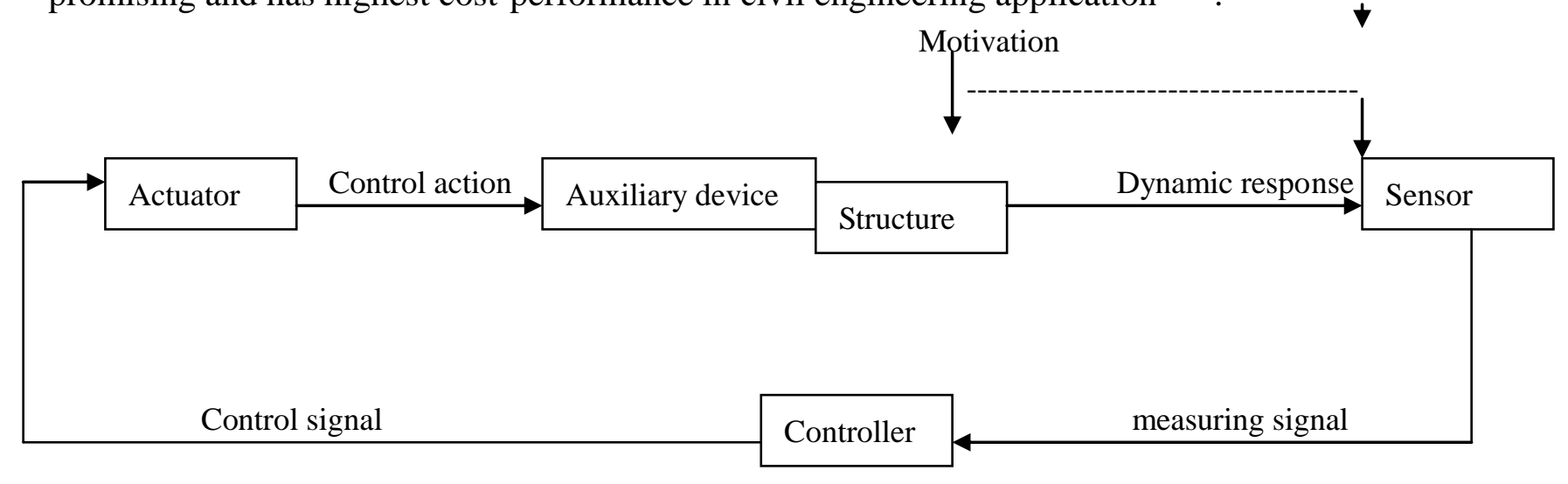

Fig. 1 Block diagram of semi-active control principle

The common two types of control devices of semi-active control are variable damping and variable stiffness:

Variable damping semi-active control system. Variable damping semi-active control system is to install variable damping device in the appropriate position of the building structure. According to the vibration response of structure, make real-time adjustment to the parameters of the variable damping device so as to provide corresponding damping force and reduce the vibration response of structure. In 1997, active variable damping control device was first adopted in US to reduce the vibration of large vehicles, and the control effect is remarkable.

Variable stiffness semi-active control system. Variable stiffness semi-active control system is to make real-time adjustment to the stiffness of building structure by the stiffness device set between layer according to seismic response, which changes the dynamic characteristics of structures and reduces earthquake damage to the building structure. After the systematic theoretical research and experimental research on variable stiffness control system made by Kobori and others, they installed active variable stiffness control device on the actual three-floor building of Tokyo, which is the first example in the world, made seismic observation and test and received good control effect.

Control device structure of semi-active control is simple and can be adjusted by itself; the energy required is much less than active structural vibration control and can get greater control. In addition, semi-active control and active control have approximate vibration damping effect and better stability, because the control force of semi-active control device is always contrary to the movement direction of building structures. The more outstanding advantage of semi-active control is the passive damping device effect through power outage when earthquake occurred. 


\section{Study on MR damper}

The parts that can make parameter adjustment of commonly adopted variable damping or variable stiffness semi-active control device mainly include mechanical parts or electric control valve, and the internal structure of these parts is very complicated. In the actual application, mechanical parts have many problems such as poor reliability, poor durability, maintenance difficulties, and inevitable delay problems. These shortcomings exist in the commonly used semi-active control device, and people have to find new semi-active control device for replacement.

ERF/MRF, which is the new-type intelligent material, is drawing close attention from academia and engineering field. ERF, short of electro-rheological fluid is composed by mother liquor that is not conductive and dielectric particles evenly distributed in mother liquor. When electric field is applied externally, the viscosity of ER will increase with the increase of electric field intensity; when the electric field intensity increases to a certain critical value, ER fluid will quickly solidify. This process is reversible and its response time is only a few milliseconds. In 1947, formal study of ERF began. However, ERF has the following disadvantages: need higher supply voltage, low shear intensity of ERF after flowing deformation, and small damping force. Therefore, ERF is not suitable for application in civil engineering structure vibration control.

MRF is short for Magneto-rheological Fluid; in the condition of external magnetic field, it can be transformed from Newton fluid into solid within millisecond, which is shown in chart 2.

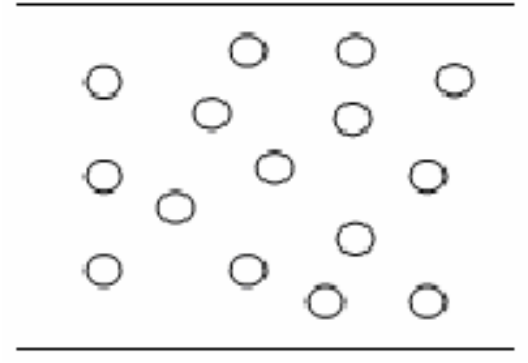

(a) without magnetic field

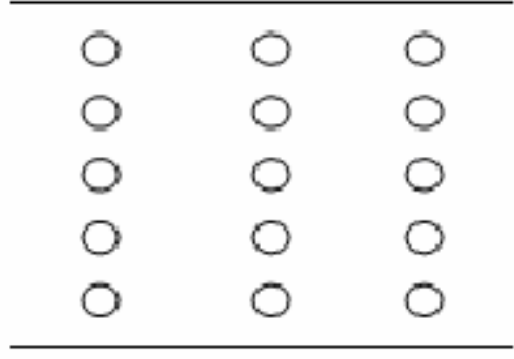

(b) in a magnetic field

Fig. 2 The working principle of magneto-rheological fluid

Working principle of magneto-rheological damper: applying electric current externally, magnetic field will be generated inside the damper; by adjusting the applied magnetic field strength and then the pressure difference at the ends of piston inside the MR damper, or adjusting the stiffness of by the MRF liquid-solid change, the control force or the damping force will be generated which has quick response and continuous change.

The three working mode of MR damper is squeeze mode, shear mode and driving mode. The working principle of three working mode is shown in chart 3: 


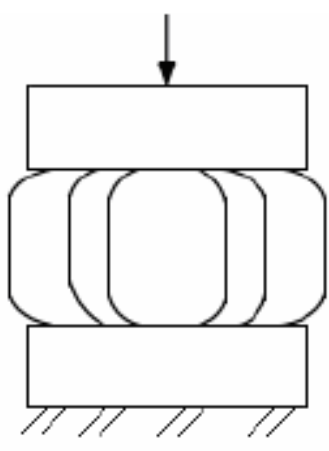

(a) Squeeze mode

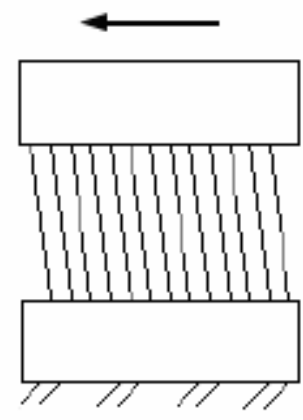

(b) Shear mode

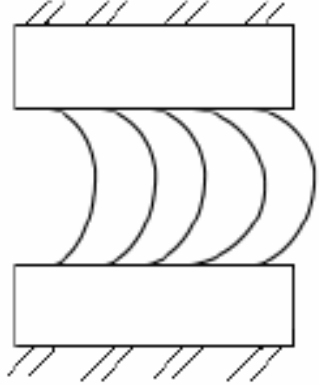

(c) Driving mode

Fig. 3 Working mode of MR damper

The mathematical model of the MR damper should be established in order to analyze the feasibility of the application of MR damper in civil engineering structure vibration control system and the need of designing semi-active control strategy. The Bingham plastic model can approximately describe the constitutive relation of magneto-rheological fluid:

$$
\tau=\tau_{y} \operatorname{sgn}(y+\eta y)
$$

In the above formula, $\tau$ is the shear force; $\tau$ is the sheared yield stress generated by magnetic field, $\gamma$ is the rate of shear strain; $\eta$ is the viscosity of MRF.

In the Bingham model, $\mathrm{x}$ is the non-zero speed of piston; for $\mathrm{x}$, damping force of MR damper can be expressed as:

$$
f=f_{c} \operatorname{sgn}(x)+c_{0} x+f_{0}
$$

\section{Radial basis function neural network (RBFNN)}

Radial Basis Function (RBF) neural network was proposed by C.Darken and J.Moody in the late 1980s, which is single layer feed-forward network of hidden layer. The activation function of RBF network hidden layer nodes is radial basis function; the characteristics of the network is of high approximation accuracy and high convergence speed and small-scale network, so it has gained wide attention. RBF network can simulate the local adjustment of human brain and acceptance domain covered each other, so there is no local minimum problem; moreover, the learning speed is extremely fast, and the network topology can be determined based on the problems. Because of these advantages of RBF neural network, it has more vitality than BP neural network, and is becoming a new type of neural network replacing BP network.

Radial basis function (RBF) neural network structure model is shown in Fig. 4, which is a special three-layer feedforward network including input layer, hidden layer and output layer. Transfer function of network hidden layer units is the radial basis function (RBF), which is to map the input vector through non-linear transform from lower dimension space to high dimension space, find the sum of weighting of hidden layer unit output, and obtain the final output. 


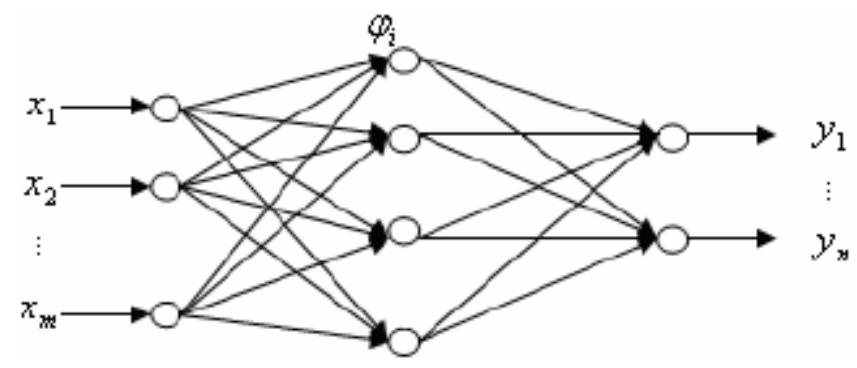

Fig. 4 RBF network structure

The hidden layer nodes of RBF network is the training sample, therefore, the number of network hidden layer nodes are the same as the number of training sample.

Suppose the number of training sample is $\mathrm{N}$, the number of nerve cell in input layer is $\mathrm{M}$, and $\mathrm{m}$ stands for one of the nerve cell; the number of nerve cell in hidden layer is $\mathrm{N}$, i stands for one of the nerve cell, and primary function $\varphi\left(x, x_{i}\right), \varphi\left(x, x_{i}\right)$ is the output of hidden layer nodes at $i$. The number of nerve cell in output layer is $\mathrm{J}$, and $\mathrm{j}$ stands for one of the arbitrary nerve cell.

When RBF network inputs the training sample $X_{k}$, the actual output of network output layer at $\mathrm{j}$ is:

$$
y_{k j}\left(X_{k}\right)=\sum_{i=1}^{N} W_{i j} \varphi\left(X_{k}, X_{i}\right), j=1,2 \cdots J
$$

Based on the analysis of structural characteristics of radial basis function (RBF) neural network, the two factors that decide the RBF network structure are: one is the number of network hidden layer nodes and the center, and the second is the connection weights between the hidden layer and output layer. Therefore, learning algorithm of commonly designed RBF network fully exploits the characteristics of the three-layer structure of RBF network. Study is generally divided into two levels: the first is to determine the number and center of network hidden layer nodes; the second is to determine the network connection weights. The linear optimization algorithm can be directly adopted in the second step, so the learning speed is improved and the local optimum is avoided; this algorithm is widely used.

\section{Conclusions}

The paper studied the semi-active control of civil engineering structure; the semi-active control research includes control device and control algorithm. This paper adopted the magneto-rheological damper as control device, and radial basis function (RBF) neural network as the control algorithm. The next time seismic response of building structure was predicted through RBF neural network; the paper designed the building structure control of RBF neural network semi-active controller under the seismic wave, and proved the effectiveness of RBF neural network. The main research work and conclusions are as follows:

The paper introduced the development of civil engineering structure vibration control, analyzed the advantages and disadvantages of passive control, active control, hybrid control and semi-active control, and summarized that the semi-active control will be the future trend of development of the civil engineering structure vibration control, which is also the research object of this article. 
This paper introduced the composition and working mechanism of the magneto-rheological fluid, and mainly introduced the working principle, working mode and dynamic model of magneto-rheological damper. The paper set up the Simulink simulation model of improved Bouc-Wen model of magneto-rheological damper, and made simulation analysis on its characteristics.

The paper made in-depth study on the basic theory and learning method of neural network, mainly studied the basic principle, the network structure and learning algorithms of radial basis function (RBF) neural network, and emphatically introduced the learning algorithms adopted in the paper. What's more, the paper studied the radial basis function (RBF) neural network toolbox of MATLAB, designed the RBF neural network structure by using the functions and commands provided by the tool kit, and trained the network.

\section{Acknowledgment}

This work was supported by Project on professional and characteristical construction of Jiangxi province 2010 (Civil Engineering) and Project on the planning and construction of disciplines in Jiangxi University of Technology (Structure Engineering)

\section{References}

[1] Yao J T P. Concept of structural control[J]. J. Struct. Engin. ASCE, 1972,16(12):1567-1574

[2] Run Weiming, Zhou Fulin, Tan Ping. The progress of civil engineering structure vibration control research [J]. World Earthquake Engineering, 1997, 13(2):8-18

[3] Cai Jing. The active control research on long-span cable-stayed bridge seismic response [D]. Chengtu: Southwest Jiaotong University, 2001

[4] Zhou Fulin. Seismic control of engineering structure [M]. Beijing: Seismological Press, 1997

[5] G.W.Housner, et al. Structural control: past, present, and future. Journal ofEngineering Mechanics, 1997, 123(9)

[6] Jiang Yicheng. Study on building structure base isolation torsional response [D] Wuhan: Huazhong University of Science and Technology, 2001

[7] Ou Jinping. Structure vibration control-active, semi-active and intelligent control [M]. Beijing Science Press, 2003:1-540

[8] Cao H, Reinhorn AM,Soong T T.Design of an active mass damper for a tall TVtower in Nanjing, China[J]. Engineering structures, 1998,20(3):134-143

[9] Tzan S R,Pantelides,P.Hybid structural control using viscoelastic dampers andactive control systems[J]. Earthquake engineering and structural dynamics, 1994,

23: $1369-1388$

[10] G. W. Housner, T. T. Soong. Second Generation ofActive Structural Controlin Civil Engineering[J]. Microcomputers in Civil Engineering.1996 (11):289-296 